\title{
The Kumaraswamy-geometric distribution
}

\author{
Alfred Akinsete ${ }^{1 * \dagger}$, Felix Famoye ${ }^{2 \dagger}$ and Carl Lee ${ }^{2 \dagger}$
}

*Correspondence:

akinsete@marshall.edu

${ }^{\dagger}$ Equal contributors

1 Department of Mathematics, Marshall University, Huntington, West Virginia 25755, USA Full list of author information is available at the end of the article

\begin{abstract}
In this paper, the Kumaraswamy-geometric distribution, which is a member of the $T$-geometric family of discrete distributions is defined and studied. Some properties of the distribution such as moments, probability generating function, hazard and quantile functions are studied. The method of maximum likelihood estimation is proposed for estimating the model parameters. Two real data sets are used to illustrate the applications of the Kumaraswamy-geometric distribution.
\end{abstract}

AMS 2010 Subject Classification: 60E05; 62E15; 62F10; 62P20

Keywords: Transformation; Moments; Entropy; Estimation

\section{Introduction}

Eugene et al. (2002) introduced the beta-generated family of univariate continuous distributions. Suppose $X$ is a random variable with cumulative distribution function (CDF) $F(x)$, the $\mathrm{CDF}$ for the beta-generated family is obtained by applying the inverse probability transformation to the beta density function. The CDF for the beta-generated family of distributions is given by

$$
G(x)=\frac{1}{B(\alpha, \beta)} \int_{0}^{F(x)} t^{\alpha-1}(1-t)^{\beta-1} d t, 0<\alpha, \beta<\infty,
$$

where $B(\alpha, \beta)=\Gamma(\alpha) \Gamma(\beta) / \Gamma(\alpha+\beta)$. The corresponding probability density function (PDF) is given by

$$
g(x)=\frac{1}{B(\alpha, \beta)}[F(x)]^{\alpha-1}[1-F(x)]^{\beta-1}\left[\frac{d}{d x} F(x)\right] .
$$

Eugene et al. (2002) used a normal random variable $X$ to define and study the betanormal distribution. Following the paper by Eugene et al. (2002), many other authors have defined and studied a number of the beta-generated distributions, using various forms of known $F(x)$. See for example, beta-Gumbel distribution by Nadarajah and Kotz (2004), beta-Weibull distribution by Famoye et al. (2005), beta-exponential distribution by Nadarajah and Kotz (2006), beta-gamma distribution by Kong et al. (2007), beta-Pareto distribution by Akinsete et al. (2008), beta-Laplace distribution by Cordeiro and Lemonte (2011), beta-generalized Weibull distribution by Singla et al. (2012), and beta-Cauchy distribution by Alshawarbeh et al. (2013), amongst others. After the paper by Jones (2009), on the tractability properties of the Kumaraswamy's distribution (Kumaraswamy 1980), Cordeiro and de Castro (2011) replaced the classical beta generator distribution with the Kumaraswamy's distribution and introduced the Kumaraswamy generated family.

(c) 2014 Akinsete et al.; licensee Springer. This is an Open Access article distributed under the terms of the Creative Commons Attribution License (http://creativecommons.org/licenses/by/4.0), which permits unrestricted use, distribution, and reproduction in any medium, provided the original work is properly credited. 
Detailed statistical properties on some Kumaraswamy generated distributions include the Kumaraswamy generalized gamma distribution by de Pascoa et al. (2011), Kumaraswamy log-logistic distribution by de Santana et al. (2012) and Kumaraswamy Gumbel distribution by Cordeiro et al. (2012). Alexander et al. (2012) replaced the beta generator distribution with the generalized beta type I distribution. The authors referred to this form as the generalized beta-generated distributions (GBGD) and the generator has three shape parameters.

The above technique of generating distributions is possible, only when the generator distributions are continuous and the random variable of the generator lies between 0 and 1. In a recent work by Alzaatreh et al. (2013b), the authors proposed a new method for generating family of distributions, referred to by the authors as the $T-X$ family, where a continuous random variable $T$ is the transformed, and any random variable $X$ is the transformer. See also Alzaatreh et al. (2012a, 2013a). These works opened a wide range of techniques for generating distributions of random variables with supports on $\mathbb{R}$. The $T-X$ family enables one to easily generate, not only the continuous distributions, but the discrete distributions as well. As a result, Alzaatreh et al. (2012b) defined and studied the $T$-geometric family, which are the discrete analogues of the distribution of the random variable $T$.

Suppose $F(x)$ denotes the CDF of any random variable $X$ and $r(t)$ denotes the PDF of a continuous random variable $T$ with support $[a, b]$. Alzaatreh et al. (2013b) gave the CDF of the $T$ - $X$ family of distributions as

$$
G(x)=\int_{a}^{W(F(x))} r(t) d t=R\{W(F(x))\}
$$

where $R(t)$ is the CDF of the random variable $T, W(F(x)) \in[a, b]$ is a non-decreasing and absolutely continuous function. Common support $[a, b]$ are $[0,1],(0, \infty)$, and $(-\infty, \infty)$. Alzaatreh et al. (2013b) studied in some details the case of a non-negative continuous random variable $T$ with support $(0, \infty)$. With this technique, it is much easier to generate any discrete distribution. If $X$ is a discrete random variable, the $T-X$ family, is a family of discrete distributions, transformed from the non-negative continuous random variable $T$. The probability mass function (PMF) of the $T-X$ family of discrete distributions may now be written as

$$
g(x)=G(x)-G(x-1)=R\{W(F(x))\}-R\{W(F(x-1))\} .
$$

The $T$-geometric family studied in Alzaatreh et al. (2012b) is a special case of (4) by defining $W(F(x))=-\ln (1-F(x))$. The rest of the paper is outlined as follows: Section 2 defines the Kumaraswamy geometric distribution (KGD). In Section 3, we discuss some properties of the distribution. In Section 4, the moments of KGD are provided, while Section 5 contains the hazard function and the Shannon entropy. In Section 6, we discuss the maximum likelihood method for estimating the parameters of the distribution. A simulation study is also discussed. Section 7 details the results of applications of the distribution to two real data sets with comparison to other distributions, and Section 8 contains some concluding remarks. 


\section{The Kumaraswamy-geometric distribution}

Following the $T$-X generalization technique by Alzaatreh et al. (2013b), we allow the transformed random variable $T$ to have the Kumaraswamy's distribution, the transformer random variable $X$ to have the geometric distribution, and $W(F(x))=F(x)$.

Kumaraswamy (1980) proposed and discussed a probability distribution for handling double-bounded random processes with varied hydrological applications. Let $T$ be a random variable with the Kumaraswamy's distribution. The PDF and CDF are defined, respectively, as

$$
\begin{aligned}
& r(t)=\alpha \beta t^{\alpha-1}\left(1-t^{\alpha}\right)^{\beta-1}, \quad 0<t<1, \quad \text { and } \\
& R(t)=1-\left(1-t^{\alpha}\right)^{\beta}, \quad 0<t<1,
\end{aligned}
$$

where both $\alpha>0$ and $\beta>0$ are the shape parameters. The beta and Kumaraswamy distributions share similar properties. For example, the Kumaraswamy's distribution, also referred to as the minimax distribution, is unimodal, uniantimodal, increasing, decreasing or constant depending on the values of its parameters. A more detailed description, background and genesis, and properties of Kumaraswamy's distribution are outlined in Jones (2009). The author highlighted several advantages of the Kumaraswamy's distribution over the beta distribution, namely; its simple normalizing constant, simple explicit formulas for the distribution and quantile functions, and simple random variate generation procedure.

The geometric distribution, also referred to as the Pascal distribution, is a special case of the negative binomial distribution. It is thought of as the discrete analogue of the continuous exponential distribution (Johnson et al. 2005). Many characterizations of the geometric distribution are analogous to the characterization of the exponential distribution. The geometric distribution has been used extensively in the literature in modeling the distribution of the lengths of waiting times. If $X$ is a random variable having the geometric distribution with parameter $p$, the PMF of $X$ may be written as

$$
P(X=x)=p q^{x}, \quad x=0,1,2, \ldots, p+q=1,
$$

where $p$ is the probability of success in a single Bernoulli trial. The CDF of the geometric distribution is given by

$$
P(X \leq x)=1-q^{x+1}, \quad x=0,1,2, \ldots
$$

The Kumaraswamy-geometric distribution (KGD) is defined by using Equation (3) with $a=0$, where the random variable $T$ has the Kumaraswamy's distribution with the $\mathrm{CDF}(6)$ and the random variable $X$ has the geometric distribution with the CDF (8). Since the random variable $T$ is defined on $(0,1)$, we use the function $W(F(x))=F(x)$ in (3) to obtain the CDF of KGD as

$$
G(x)=\int_{0}^{F(x)} r(t) d t=R(F(x))=1-\left[1-\left(1-q^{x+1}\right)^{\alpha}\right]^{\beta}, \quad x=0,1,2, \ldots
$$

The corresponding PMF for the KGD now becomes

$$
g(x)=\left[1-\left(1-q^{x}\right)^{\alpha}\right]^{\beta}-\left[1-\left(1-q^{x+1}\right)^{\alpha}\right]^{\beta}, \quad x=0,1,2, \ldots, \alpha>0, \beta>0,
$$


by using Equation (4). Thus, a random variable $X$ having the PMF expressed in Equation (10) is said to follow the Kumaraswamy-geometric distribution with parameters $\alpha, \beta$ and $q$, or simply $X \sim \operatorname{KGD}(\alpha, \beta, q)$. One can show that the PMF in Equation (10) satisfies $\sum_{0}^{\infty} g(x)=1$ by telescopic cancellation.

It is interesting to note that the KGD can be generated from a different random variable $T$ and a different $W(F(x))$ function. Suppose a random variable $Y$ follows the Kumaraswamy's distribution in (5), then its PDF is

$$
f(y)=\alpha \beta y^{\alpha-1}\left(1-y^{\alpha}\right)^{\beta-1}, \quad 0<y<1 .
$$

Suppose we define a new random variable as $T=-\ln (1-Y)$. By using the transformation technique, the PDF of $T$ is given by

$$
f(t)=\alpha \beta e^{-t}\left(1-e^{-t}\right)^{\alpha-1}\left[1-\left(1-e^{-t}\right)^{\alpha}\right]^{\beta-1}, \quad t>0 .
$$

The corresponding CDF is given by

$$
F(t)=1-\left[1-\left(1-e^{-t}\right)^{\alpha}\right]^{\beta}, \quad t>0 .
$$

A random variable $T$ with the CDF in (12) will be called the log-Kumaraswamy's distribution (LKD). We are unable to find any reference to this distribution in the literature. However, it is a special case of the log-exponentiated Kumaraswamy distribution studied by Lemonte et al. (2013). By using the LKD and the $T$ - $X$ distribution by Alzaatreh et al. (2013b), we can define the log-Kumaraswamy-geometric distribution (LKGD) by using Equation (3), where $T$ follows the LKD, $X$ follows the geometric distribution and $W(F(x))=-\ln (1-F(x))$. By using $1-F(x)=q^{x+1}$ and $-\ln (1-F(x))=-\ln q^{x+1}$, the probability mass function of LKGD can be obtained as

$$
g(x)=G(x)-G(x-1)=R\left[-\ln q^{x+1}\right]-R\left[-\ln q^{x}\right]=\left[1-\left(1-q^{x}\right)^{\alpha}\right]^{\beta}-\left[1-\left(1-q^{x+1}\right)^{\alpha}\right]^{\beta},
$$

which is the same as the KGD in (10) defined by using Kumaraswamy's and geometric distributions. The LKGD, and hence the KGD, is the discrete analogue of log-Kumaraswamy's distribution.

\section{Special cases of KGD}

The following are special cases of KGD:

(a) When $\alpha=\beta=1$, the KGD in (10) reduces to the geometric distribution in (7) with parameter $p$.

(b) When $\alpha=1$, the KGD with parameters $\alpha, \beta$ and $q$ reduces to the geometric distribution with parameter $p_{*}$, where $p_{*}=1-q^{\beta}$.

(c) When $\beta=1$, the KGD reduces to the exponentiated-exponential-geometric distribution (EEGD) discussed in Alzaatreh et al. (2012b).

It is easy to verify that $\lim _{x \rightarrow \infty} G(x)=1$. The plots of the PMF of the KGD for various values of $\alpha, \beta$ and $q$ are given in Figure 1 .

\section{Some properties of Kumaraswamy-geometric distribution}

Suppose $X$ follows the KGD with CDF $G(x)$ in (9). The quantile function $X_{*}(=Q(U), 0<$ $U<1)$ of KGD is the inverse of the cumulative distribution. That is,

$$
X_{*}=Q(U)=(\log q)^{-1} \log \left\{1-\left[1-(1-U)^{1 / \beta}\right]^{1 / \alpha}\right\},
$$




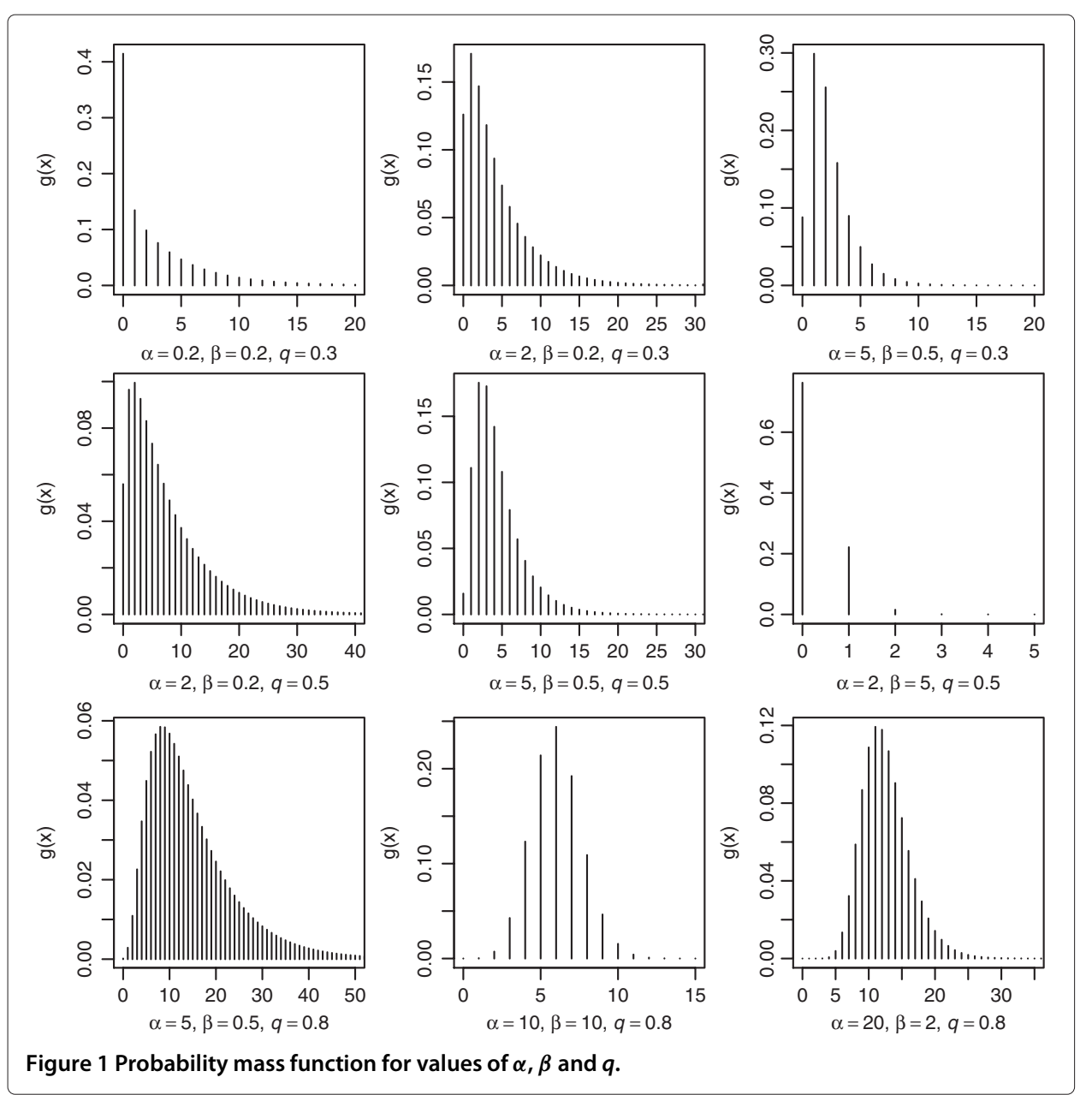

where $U$ has a uniform distribution with support on $(0,1)$. Equation (14) can be used to simulate the Kumaraswamy-geometric random variable. First, simulate a random variable $U$ and compute the value of $X_{*}$ in (14), which is not necessarily an integer. The Kumaraswamy-geometric random variate $X$ is the largest integer $\leq X_{*}$, which can be denoted by $\left[X_{*}\right]$.

Transformation: The relationship between the KGD and the Kumaraswamy's, exponential, exponentiated-exponential, Pareto, Weibull, Rayleigh, and the logistic distributions are given in the following lemma.

Lemma 1. Suppose $[v]$ denotes the largest integer less than or equal to the quantity $v$.

(a) If $Y$ has Kumaraswamy's distribution with parameters $\alpha$ and $\beta$, then the distribution of $X=\left[\log _{q}(1-Y)\right]$ is $K G D$.

(b) If $Y$ is standard exponential, then $X=\left[\log _{q}\left\{1-\left(1-e^{-Y / \beta}\right)^{1 / \alpha}\right\}\right]$ has KGD.

(c) If $Y$ follows an exponentiated-exponential distribution with scale parameter $\lambda$ and index parameter $c$, then $X=\left[\log _{q}\left\{1-\left[1-\left(1-e^{-\lambda Y}\right)^{c / \beta}\right]^{1 / \alpha}\right\}\right]$ has KGD. 
(d) If the random variable $Y$ has a Pareto distribution with parameters $\theta, k$ and CDF

$$
F(y)=1-\left(\frac{\theta}{\theta+y}\right)^{k} \text {, then } X=\left[\log _{q}\left\{1-\left(1-\left(\frac{\theta}{\theta+Y}\right)^{k / \beta}\right)^{1 / \alpha}\right\}\right] \text { has KGD. }
$$

(e) If the random variable $Y$ has a Weibull distribution with $F(y)=1-\exp \left\{-(y / \gamma)^{c}\right\}$ as $C D F$, then $X=\left[\log _{q}\left\{1-\left(1-\exp \left[-(Y / \gamma)^{c} / \beta\right]\right)^{1 / \alpha}\right\}\right]$ has $K G D$.

(f) If the random variable $Y$ has a Rayleigh distribution with $F(y)=1-\exp \left[\frac{-y^{2}}{2 b^{2}}\right]$ as $C D F$, then $X=\left[\log _{q}\left\{1-\left(1-\exp \left[\frac{-Y^{2}}{2 b^{2} \beta}\right]\right)^{1 / \alpha}\right\}\right]$ has KGD.

(g) If $Y$ is a logistic random variable with $F(y)=[1+\exp (\{-(y-a) / b\})]^{-1}$ as $C D F$, then $X=\left[\log _{q}\left\{1-\left(1-[1+\exp \{-(Y-a) / b\}]^{-1 / \beta}\right)^{1 / \alpha}\right\}\right]$ has KGD.

Proof. By using the transformation technique, it is easy to show that the random variable $X$ has KGD as given in (10). We will show the result for part (a). Let $R$ be the CDF of the Kumaraswamy's distribution.

$$
\begin{aligned}
P(X=x) & =P\left(\left[\log _{q}(1-Y)\right]=x\right)=P\left(x \leq \log _{q}(1-Y)<x+1\right) \\
& =P\left(1-q^{x} \leq Y<1-q^{x+1}\right)=R\left(1-q^{x+1}\right)-R\left(1-q^{x}\right) \\
& =\left\{1-\left(1-q^{x}\right)^{\alpha}\right\}^{\beta}-\left\{1-\left(1-q^{x+1}\right)^{\alpha}\right\}^{\beta},
\end{aligned}
$$

which is the PMF of the KGD in (10).

In general, if we have a continuous random variable $Y$ and its CDF is $F(y)$, then $X=$ $\left[\log _{q}\left\{1-\left(1-F^{1 / \beta}(Y)\right)^{1 / \alpha}\right\}\right]$ has KGD.

Limiting behavior: As $x \rightarrow \infty, \lim _{x \rightarrow \infty} g(x)=0$. Also, as $x \rightarrow 0, \lim _{x \rightarrow 0} g(x)=1-[1-$ $\left.(1-q)^{\alpha}\right]^{\beta}$. This limit becomes 0 if $q \rightarrow 1$ and/or $\alpha \rightarrow \infty$. Thus, the distribution starts with probability zero or a constant probability as evident from Figure 1.

Mode of the KGD: Since the KGD is also LKGD, a T-geometric distribution, we use Lemma 2 in Alzaatreh et al. (2012b), which states that a $T$-geometric distribution has a reversed J-shape if the distribution of the random variable $T$ has a reversed J-shape. We only need to show when the distribution of log-Kumaraswamy distribution has a reversed J-shape.

On taking the first derivative of (11) with respect to $t$, we obtain

$$
f^{\prime}(t)=\left[\alpha e^{-t}-1+\left(1-e^{-t}\right)^{\alpha}-\alpha \beta e^{-t}\left(1-e^{-t}\right)^{\alpha}\right] V(t)=Q(t) V(t),
$$

where $V(t)=\alpha \beta e^{-t}\left(1-e^{-t}\right)^{\alpha-2}\left[1-\left(1-e^{-t}\right)^{\alpha}\right]^{\beta-2}$ is positive. For $\beta \geq 1$ and $\alpha \leq 1$, it is straight forward to show that $Q(t) \leq 0$. For $\beta<1$ and $\alpha \leq 1$, the function $Q(t)$ is an increasing function of $t$. It is not difficult to show that $\lim _{t \rightarrow 0} Q(t)=\alpha-1 \leq 0$ and $\lim _{t \rightarrow \infty} Q(t)=0$. Thus, for $\alpha \leq 1$ and any value of $\beta$ and $q, Q(t) \leq 0$ and so the PDF of the log-Kumaraswamy distribution is monotonically decreasing or has a reversed J-shape. Hence, the KGD has a reversed J-shape and a unique mode at $x=0$ when $\alpha \leq 1$.

When $\alpha>1$, it is not easy to show that the KGD is unimodal. However, through numerical analysis of the behavior of the PMF, and its plots in Figure 1 for various values of $\beta$ and $q$, we observe, that for values of $\alpha>1$, the KGD is concave down or has a reversed $\mathrm{J}$-shape with a unique mode. 


\section{Moments}

Using Equation (10), the $r^{\text {th }}$ raw moment is given by

$$
\begin{aligned}
E\left(X^{r}\right)=\mu_{r}^{\prime} & =\sum_{x=1}^{\infty} x^{r}\left\{1-\left(1-q^{x}\right)^{\alpha}\right\}^{\beta}-\sum_{x=1}^{\infty} x^{r}\left\{1-\left(1-q^{x+1}\right)^{\alpha}\right\}^{\beta} \\
& =\sum_{x=1}^{\infty} x^{r}\left[\left(\sum_{i=1}^{\infty}(-1)^{i-1}\left(\begin{array}{c}
\alpha \\
i
\end{array}\right) q^{x i}\right)^{\beta}-\left(\sum_{i=1}^{\infty}(-1)^{i-1}\left(\begin{array}{c}
\alpha \\
i
\end{array}\right) q^{(x+1) i}\right)^{\beta}\right]
\end{aligned}
$$

The two inner summations terminate at $\alpha$, if $\alpha$ is a positive integer. When $\beta=1$ in the above, we have,

$$
\mu_{r}^{\prime}=\sum_{x=1}^{\infty} x^{r} \sum_{i=1}^{\alpha}(-1)^{i-1}\left(\begin{array}{c}
\alpha \\
i
\end{array}\right) q^{x i}\left(1-q^{i}\right), \text { for } \alpha \in \mathcal{Z}^{+} .
$$

In particular, let $r=1$ and $\alpha=1$, the expression for the first moment, or the mean of the KGD may be written as,

$$
E(X)=\mu_{1}^{\prime}=q(1-q) \sum_{x=1}^{\infty} x q^{x-1}=\frac{q}{p},
$$

which is the mean of the geometric distribution, a special case of KGD.

We discuss in what follows, an alternative approach of expressing the PMF of the KGD in Equation (10).

$$
\begin{aligned}
g(x) & =\left\{1-\left(1-q^{x}\right)^{\alpha}\right\}^{\beta}-\left\{1-\left(1-q^{x+1}\right)^{\alpha}\right\}^{\beta} \\
& =\sum_{i=0}^{\infty}(-1)^{i}\left(\begin{array}{c}
\beta \\
i
\end{array}\right)\left(1-q^{x}\right)^{\alpha i}-\sum_{i=0}^{\infty}(-1)^{i}\left(\begin{array}{c}
\beta \\
i
\end{array}\right)\left(1-q^{x+1}\right)^{\alpha i} \\
& =\sum_{i=0}^{\infty}(-1)^{i}\left(\begin{array}{c}
\beta \\
i
\end{array}\right) \sum_{j=0}^{\infty}(-1)^{j}\left(\begin{array}{c}
\alpha i \\
j
\end{array}\right) q^{x j}-\sum_{i=0}^{\infty}(-1)^{i}\left(\begin{array}{c}
\beta \\
i
\end{array}\right) \sum_{j=0}^{\infty}(-1)^{j}\left(\begin{array}{c}
\alpha i \\
j
\end{array}\right) q^{(x+1) j} \\
& =\sum_{i=1}^{\infty} \sum_{j=1}^{\infty}(-1)^{i+j}\left(\begin{array}{c}
\beta \\
i
\end{array}\right)\left(\begin{array}{c}
\alpha i \\
j
\end{array}\right)\left(1-q^{j}\right) q^{x j} .
\end{aligned}
$$

Using Equation (16), it is now easy to write the expressions for the moment, moment generating function, and probability generating function for the KGD respectively as follows:

$$
\begin{aligned}
& \mu_{r}^{\prime}=\sum_{x=0}^{\infty} \sum_{i=1}^{\infty} \sum_{j=1}^{\infty}(-1)^{i+j}\left(\begin{array}{c}
\beta \\
i
\end{array}\right)\left(\begin{array}{c}
\alpha i \\
j
\end{array}\right)\left(1-q^{j}\right) x^{r}\left(q^{j}\right)^{x} ; \quad\left|q^{j}\right|<1, \\
& M(t)=\sum_{x=0}^{\infty} \sum_{i=1}^{\infty} \sum_{j=1}^{\infty}(-1)^{i+j}\left(\begin{array}{c}
\beta \\
i
\end{array}\right)\left(\begin{array}{c}
\alpha i \\
j
\end{array}\right)\left(1-q^{j}\right)\left(e^{t} q^{j}\right)^{x} ; \quad\left|e^{t} q^{j}\right|<1, \\
& \varphi(t)=\sum_{x=0}^{\infty} \sum_{i=1}^{\infty} \sum_{j=1}^{\infty}(-1)^{i+j}\left(\begin{array}{c}
\beta \\
i
\end{array}\right)\left(\begin{array}{c}
\alpha i \\
j
\end{array}\right)\left(1-q^{j}\right)\left(t q^{j}\right)^{x} ; \quad\left|t q^{j}\right|<1 .
\end{aligned}
$$

Equation (17) is equivalent to the series $\sum_{x=0}^{\infty} x^{r} g(x)$, where $g(x)$ is given by (10). Observe that the series is absolutely convergent by using the ratio test and hence the 
series in (17) is absolutely convergent. Thus, interchanging the order of summation has no effect. Using Equation (17), the $r^{\text {th }}$ moment may be written as

$$
\begin{aligned}
E\left(X^{r}\right)=\mu_{r}^{\prime} & =\sum_{x=0}^{\infty} \sum_{i=1}^{\infty} \sum_{j=1}^{\infty}(-1)^{i+j}\left(\begin{array}{c}
\beta \\
i
\end{array}\right)\left(\begin{array}{c}
\alpha i \\
j
\end{array}\right)\left(1-q^{j}\right)\left(q^{j}\right)^{x} x^{r} \\
& =\sum_{i=1}^{\infty} \sum_{j=1}^{\infty}(-1)^{i+j}\left(\begin{array}{c}
\beta \\
i
\end{array}\right)\left(\begin{array}{c}
\alpha i \\
j
\end{array}\right)\left(1-q^{j}\right) \sum_{x=1}^{\infty}\left(q^{j}\right)^{x} x^{r} \\
& =\sum_{i=1}^{\infty} \sum_{j=1}^{\infty}(-1)^{i+j} \frac{\beta^{(i)}}{i !} \frac{(\alpha i)^{(j)}}{j !}\left(1-q^{j}\right) L_{-r}\left(q^{j}\right),
\end{aligned}
$$

where $\beta^{(i)}=\beta(\beta-1)(\beta-2) \cdots(\beta-i+1)$, and similarly for $(\alpha i)^{(j)}$. Also,

$$
L_{-r}(u)=\sum_{k=1}^{\infty} \frac{u^{k}}{k^{-r}}, \quad u=q^{j},
$$

is the polylogarithm function, (http://mathworld.wolfram.com/Polylogarithm.html).

Expressions for the first few moments are thus:

$$
\begin{aligned}
& \mu_{1}^{\prime}=\sum_{i=1}^{\infty} \sum_{j=1}^{\infty}(-1)^{i+j} \frac{\beta^{(i)}}{i !} \frac{(\alpha i)^{(j)}}{j !} \frac{q^{j}}{1-q^{j}}, \\
& \mu_{2}^{\prime}=\sum_{i=1}^{\infty} \sum_{j=1}^{\infty}(-1)^{i+j} \frac{\beta^{(i)}}{i !} \frac{(\alpha i)^{(j)}}{j !} \frac{q^{j}\left(1+q^{j}\right)}{\left(1-q^{j}\right)^{2}}, \\
& \mu_{3}^{\prime}=\sum_{i=1}^{\infty} \sum_{j=1}^{\infty}(-1)^{i+j} \frac{\beta^{(i)}}{i !} \frac{(\alpha i)^{(j)}}{j !} \frac{q^{j}\left(1+4 q^{j}+q^{2 j}\right)}{\left(1-q^{j}\right)^{3}}, \\
& \mu_{4}^{\prime}=\sum_{i=1}^{\infty} \sum_{j=1}^{\infty}(-1)^{i+j} \frac{\beta^{(i)}}{i !} \frac{(\alpha i)^{(j)}}{j !} \frac{q^{j}\left(1+q^{j}\right)\left(1+10 q^{j}+q^{2 j}\right)}{\left(1-q^{j}\right)^{4}} .
\end{aligned}
$$

The expression for the variance may be written as

$$
\sigma^{2}=\sum_{i=1}^{\infty} \sum_{j=1}^{\infty}(-1)^{i+j} \frac{\beta^{(i)}}{i !} \frac{(\alpha i)^{(j)}}{j !} \frac{q^{j}\left(1+q^{j}\right)}{\left(1-q^{j}\right)^{2}}-\left(\sum_{i=1}^{\infty} \sum_{j=1}^{\infty}(-1)^{i+j} \frac{\beta^{(i)}}{i !} \frac{(\alpha i)^{(j)}}{j !} \frac{q^{j}}{1-q^{j}}\right)^{2} .
$$

Expressions for the skewness and kurtosis for the KGD may be obtained by combining appropriate expressions in Equations (20), (21), (22), and (23). In the particular case for which $\alpha=1=\beta$, the expressions for the central moments of the geometric distribution are as follows:

$$
\mu_{1}=\mu_{1}^{\prime}=\frac{q}{p}, \quad \mu_{2}=\sigma^{2}=\frac{q}{p^{2}}, \quad \mu_{3}=\frac{q(1+q)}{p^{3}}, \quad \mu_{4}=\frac{q\left(p^{2}+9 q\right)}{p^{4}} .
$$

The results for this special case may be found in standard textbooks on probability. See for example, Zwillinger and Kokoska (2000).

Both the moment generating function $(M(t))$ and the probability generating function $(\varphi(t))$ can be simplified further. In the case of $\varphi(t)$, we have

$$
\varphi(t)=\sum_{i=1}^{\infty} \sum_{j=1}^{\infty}(-1)^{i+j}\left(\begin{array}{c}
\beta \\
i
\end{array}\right)\left(\begin{array}{c}
\alpha i \\
j
\end{array}\right)\left(1-q^{j}\right) \sum_{x=0}^{\infty}\left(q^{j}\right)^{x} t^{x}, \quad\left|t q^{j}\right|<1 \quad \forall j .
$$


After further simplification, the above reduces to,

$$
\varphi(t)=\sum_{i=1}^{\infty} \sum_{j=1}^{\infty}(-1)^{i+j}\left(\begin{array}{c}
\beta \\
i
\end{array}\right)\left(\begin{array}{c}
\alpha i \\
j
\end{array}\right) \frac{\left(1-q^{j}\right)}{\left(1-t q^{j}\right)} .
$$

By letting

$$
A(\alpha, \beta \mid i, j)=\sum_{i=1}^{\infty} \sum_{j=1}^{\infty}(-1)^{i+j}\left(\begin{array}{c}
\beta \\
i
\end{array}\right)\left(\begin{array}{c}
\alpha i \\
j
\end{array}\right),
$$

the first two factorial moments may be expressed as

$$
\begin{aligned}
& \varphi^{\prime}(t=1)=\mu_{[1]}=E(X)=A(\alpha, \beta \mid i, j) \frac{q^{j}}{1-q^{j}} \\
& \varphi^{\prime \prime}(t=1)=\mu_{[2]}=E(X(X-1))=A(\alpha, \beta \mid i, j) \frac{2 q^{2 j}}{\left(1-q^{j}\right)^{2}} .
\end{aligned}
$$

In general,

$$
\varphi^{(m)}(t)=A(\alpha, \beta \mid i, j) \frac{m !\left(1-q^{j}\right) q^{m j}}{\left(1-t q^{j}\right)^{m+1}},
$$

which reduces to the result in Alzaatreh et al. (2012b) when $\beta=1$.

Through numerical computation, we obtain the mode, the mean, the standard deviation (SD), the skewness and the kurtosis of the KGD. The values of $\alpha$ and $\beta$ for the numerical computation are from 0.2 to 10 at an increment of 0.1 , while the values of $q$ are from 0.2 to 0.9 at an increment of 0.1 . For brevity, we report the mode, the mean and the standard deviation in Table 1 and the skewness and kurtosis in Table 2 for some values of $q, \beta$ and $\alpha$. From the numerical computation, the mean, mode and standard deviation are increasing functions of $q$. From Table 1 , the mean, mode and standard deviation are decreasing functions of $\beta$ but increasing functions of $\alpha$. For $\alpha \leq 1$, the skewness and kurtosis are decreasing functions of $q$ but increasing functions of $\beta$. For $\alpha>1$, the skewness and kurtosis first decrease and then increase as both $q$ and $\beta$ increase. The skewness and kurtosis are decreasing functions of $\alpha$. Some of these observations can be seen in Table 2 while others are from the numerical computation. Instead of Tables 1 and 2, contour plots may be used to present the results in the tables. However, it becomes difficult to see the patterns described above.

\section{Hazard rate and Shannon entropy}

The hazard rate function is defined as

$$
h(x)=\frac{g(x)}{1-G(x)},
$$

where $G(x)=\sum_{y=0}^{x} g(y)$. For the KGD, we have, after substituting expressions for the PMF and CDF (Equations (10) and (9)),

$$
h(x)=\left(\frac{1-\left(1-q^{x}\right)^{\alpha}}{1-\left(1-q^{x+1}\right)^{\alpha}}\right)^{\beta}-1 .
$$

The asymptotic behaviors of the hazard function are such that,

$$
\lim _{x \rightarrow 0} h(x)=\left(1-p^{\alpha}\right)^{-\beta}-1=L_{1},
$$

and in particular, $\lim _{x \rightarrow 0} h(x ; \alpha=1=\beta)=p / q=1 / \mathrm{E}(X)$. Also, $\lim _{x \rightarrow \infty} h(x)=q^{-\beta}-$ $1=L_{2}$, after using the L'Hôspital's rule. This result generalizes the limiting behavior of 
Table 1 Mode, mean and standard deviation (SD) of KGD for some values of $\alpha, \beta$ and $q$

\begin{tabular}{|c|c|c|c|c|c|c|c|c|c|c|}
\hline \multirow[b]{2}{*}{$\alpha$} & \multirow[b]{2}{*}{$\beta$} & \multicolumn{3}{|c|}{$q=0.4$} & \multicolumn{3}{|c|}{$q=0.6$} & \multicolumn{3}{|c|}{$q=0.8$} \\
\hline & & Mode & Mean & SD & Mode & Mean & SD & Mode & Mean & SD \\
\hline \multirow[t]{6}{*}{0.4} & 0.4 & 0 & 1.60 & 2.48 & 0 & 3.16 & 4.51 & 0 & 7.74 & 10.40 \\
\hline & 0.6 & 0 & 0.83 & 1.52 & 0 & 1.72 & 2.81 & 0 & 4.39 & 6.54 \\
\hline & 0.8 & 0 & 0.48 & 1.04 & 0 & 1.06 & 1.96 & 0 & 2.82 & 4.60 \\
\hline & 1.5 & 0 & 0.10 & 0.39 & 0 & 0.29 & 0.79 & 0 & 0.92 & 1.96 \\
\hline & 2.0 & 0 & 0.04 & 0.22 & 0 & 0.13 & 0.49 & 0 & 0.49 & 1.26 \\
\hline & 4.0 & 0 & 0.001 & 0.04 & 0 & 0.01 & 0.11 & 0 & 0.07 & 0.35 \\
\hline \multirow[t]{6}{*}{0.6} & 0.4 & 0 & 1.87 & 2.59 & 0 & 3.67 & 4.69 & 0 & 8.97 & 10.80 \\
\hline & 0.6 & 0 & 1.04 & 1.65 & 0 & 2.14 & 3.02 & 0 & 5.43 & 6.99 \\
\hline & 0.8 & 0 & 0.64 & 1.17 & 0 & 1.41 & 2.18 & 0 & 3.71 & 5.08 \\
\hline & 1.5 & 0 & 0.18 & 0.51 & 0 & 0.48 & 1.00 & 0 & 1.48 & 2.42 \\
\hline & 2.0 & 0 & 0.08 & 0.32 & 0 & 0.26 & 0.67 & 0 & 0.91 & 1.67 \\
\hline & 4.0 & 0 & 0.005 & 0.07 & 0 & 0.04 & 0.20 & 0 & 0.21 & 0.62 \\
\hline \multirow[t]{6}{*}{0.8} & 0.4 & 0 & 2.08 & 2.66 & 0 & 4.08 & 4.81 & 0 & 9.93 & 11.04 \\
\hline & 0.6 & 0 & 1.21 & 1.74 & 0 & 2.49 & 3.16 & 0 & 6.27 & 7.27 \\
\hline & 0.8 & 0 & 0.79 & 1.27 & 0 & 1.71 & 2.33 & 0 & 4.47 & 5.38 \\
\hline & 1.5 & 0 & 0.26 & 0.60 & 0 & 0.68 & 1.16 & 0 & 2.01 & 2.74 \\
\hline & 2.0 & 0 & 0.13 & 0.40 & 0 & 0.41 & 0.82 & 0 & 1.35 & 1.99 \\
\hline & 4.0 & 0 & 0.01 & 0.12 & 0 & 0.08 & 0.31 & 0 & 0.43 & 0.87 \\
\hline \multirow[t]{6}{*}{1.5} & 0.4 & 1 & 2.61 & 2.79 & 1 & 5.06 & 5.00 & 3 & 12.22 & 11.44 \\
\hline & 0.6 & 0 & 1.68 & 1.89 & 1 & 3.39 & 3.39 & 2 & 8.38 & 7.76 \\
\hline & 0.8 & 0 & 1.21 & 1.44 & 1 & 2.54 & 2.59 & 2 & 6.43 & 5.92 \\
\hline & 1.5 & 0 & 0.54 & 0.81 & 0 & 1.31 & 1.47 & 1 & 3.61 & 3.35 \\
\hline & 2.0 & 0 & 0.35 & 0.62 & 0 & 0.95 & 1.14 & 1 & 2.77 & 2.61 \\
\hline & 4.0 & 0 & 0.08 & 0.29 & 0 & 0.38 & 0.63 & 0 & 1.42 & 1.46 \\
\hline \multirow[t]{6}{*}{2.0} & 0.4 & 1 & 2.87 & 2.83 & 2 & 5.55 & 5.06 & 4 & 13.35 & 11.57 \\
\hline & 0.6 & 1 & 1.93 & 1.94 & 1 & 3.85 & 3.47 & 4 & 9.45 & 7.92 \\
\hline & 0.8 & 1 & 1.44 & 1.50 & 1 & 2.97 & 2.68 & 3 & 7.45 & 6.10 \\
\hline & 1.5 & 0 & 0.73 & 0.89 & 1 & 1.69 & 1.58 & 2 & 4.51 & 3.57 \\
\hline & 2.0 & 0 & 0.51 & 0.71 & 1 & 1.30 & 1.26 & 2 & 3.61 & 2.83 \\
\hline & 4.0 & 0 & 0.18 & 0.40 & 0 & 0.65 & 0.77 & 1 & 2.11 & 1.69 \\
\hline \multirow[t]{6}{*}{4.0} & 0.4 & 2 & 3.56 & 2.89 & 3 & 6.79 & 5.16 & 8 & 16.19 & 11.79 \\
\hline & 0.6 & 1 & 2.59 & 2.02 & 3 & 5.05 & 3.59 & 7 & 12.21 & 8.19 \\
\hline & 0.8 & 1 & 2.09 & 1.59 & 2 & 4.14 & 2.81 & 6 & 10.13 & 6.41 \\
\hline & 1.5 & 1 & 1.32 & 1.01 & 2 & 2.77 & 1.74 & 5 & 7.00 & 3.94 \\
\hline & 2.0 & 1 & 1.08 & 0.84 & 2 & 2.34 & 1.43 & 4 & 5.99 & 3.23 \\
\hline & 4.0 & 1 & 0.64 & 0.61 & 1 & 1.57 & 0.96 & 3 & 4.24 & 2.10 \\
\hline \multirow[t]{6}{*}{6.0} & 0.4 & 2 & 3.99 & 2.90 & 4 & 7.55 & 5.19 & 10 & 17.92 & 11.87 \\
\hline & 0.6 & 2 & 3.01 & 2.04 & 4 & 5.79 & 3.63 & 9 & 13.90 & 8.29 \\
\hline & 0.8 & 2 & 2.50 & 1.61 & 3 & 4.87 & 2.86 & 8 & 11.80 & 6.53 \\
\hline & 1.5 & 1 & 1.72 & 1.03 & 3 & 3.47 & 1.80 & 7 & 8.59 & 4.08 \\
\hline & 2.0 & 1 & 1.46 & 0.87 & 2 & 3.02 & 1.50 & 6 & 7.55 & 3.38 \\
\hline & 4.0 & 1 & 1.02 & 0.62 & 2 & 2.21 & 1.02 & 5 & 5.71 & 2.27 \\
\hline
\end{tabular}

the hazard rate function for the EEGD discussed in Alzaatreh et al. (2012b). Observe that $L_{1}>L_{2}$ when $\alpha<1$. For $\alpha<1$, we check the behavior of $h(x)$. The function $h(x)$ is monotonically decreasing when $\alpha<1$ if $h(x) \geq h(x+1)$ for all $x$. When $\alpha=1$, observe that $h(x)$ is a constant. For values of $\alpha<1$, we numerically evaluate $d(x)=$ $h(x)-h(x+1)$ for $\alpha$ and $q$ from 0.1 to 0.9 at an increment of 0.1 . All the values are 
Table 2 Skewness and kurtosis of KGD for some values of $\alpha, \beta$ and $q$

\begin{tabular}{|c|c|c|c|c|c|c|c|}
\hline \multirow[b]{2}{*}{$\alpha$} & \multirow[b]{2}{*}{$\beta$} & \multicolumn{2}{|c|}{$q=0.4$} & \multicolumn{2}{|c|}{$q=0.6$} & \multicolumn{2}{|c|}{$q=0.8$} \\
\hline & & Skewness & Kurtosis & Skewness & Kurtosis & Skewness & Kurtosis \\
\hline \multirow[t]{6}{*}{0.4} & 0.4 & 2.4529 & 8.4883 & 2.3789 & 8.0612 & 2.3360 & 7.8215 \\
\hline & 0.6 & 2.8022 & 10.920 & 2.6534 & 9.9332 & 2.5656 & 9.3787 \\
\hline & 0.8 & 3.1918 & 14.035 & 2.9430 & 12.172 & 2.7954 & 11.133 \\
\hline & 1.5 & 4.9662 & 32.736 & 4.0792 & 23.245 & 3.5894 & 18.553 \\
\hline & 2.0 & 6.8722 & 60.013 & 5.0708 & 35.519 & 4.1724 & 25.214 \\
\hline & 4.0 & 30.524 & 990.63 & 12.671 & 196.82 & 7.1320 & 72.567 \\
\hline \multirow[t]{6}{*}{0.6} & 0.4 & 2.2480 & 7.2663 & 2.1956 & 7.0004 & 2.1684 & 6.8666 \\
\hline & 0.6 & 2.4465 & 8.4649 & 2.3387 & 7.8659 & 2.2823 & 7.5649 \\
\hline & 0.8 & 2.6694 & 9.9363 & 2.4883 & 8.8459 & 2.3932 & 8.3020 \\
\hline & 1.5 & 3.6723 & 17.839 & 3.0582 & 13.119 & 2.7529 & 10.999 \\
\hline & 2.0 & 4.6998 & 27.856 & 3.5337 & 17.209 & 2.9979 & 13.043 \\
\hline & 4.0 & 14.964 & 238.38 & 6.6621 & 54.450 & 4.0916 & 23.691 \\
\hline \multirow[t]{6}{*}{0.8} & 0.4 & 2.1208 & 6.5743 & 2.0847 & 6.4108 & 2.0685 & 6.3383 \\
\hline & 0.6 & 2.2269 & 7.1361 & 2.1498 & 6.7675 & 2.1151 & 6.6063 \\
\hline & 0.8 & 2.3522 & 7.8283 & 2.2198 & 7.1642 & 2.1603 & 6.8772 \\
\hline & 1.5 & 2.9553 & 11.535 & 2.5010 & 8.8497 & 2.3055 & 7.8006 \\
\hline & 2.0 & 3.5861 & 16.072 & 2.7466 & 10.413 & 2.4048 & 8.4499 \\
\hline & 4.0 & 9.2202 & 90.141 & 4.3332 & 22.881 & 2.8543 & 11.487 \\
\hline \multirow[t]{6}{*}{1.5} & 0.4 & 1.9024 & 5.5182 & 1.9015 & 5.5273 & 1.9038 & 5.5384 \\
\hline & 0.6 & 1.8485 & 5.2051 & 1.8400 & 5.2063 & 1.8433 & 5.2269 \\
\hline & 0.8 & 1.8105 & 4.9322 & 1.7862 & 4.9087 & 1.7894 & 4.9374 \\
\hline & 1.5 & 1.8191 & 4.4671 & 1.6645 & 4.1552 & 1.6529 & 4.1894 \\
\hline & 2.0 & 1.9494 & 4.6197 & 1.6281 & 3.8307 & 1.5890 & 3.8375 \\
\hline & 4.0 & 3.3608 & 11.0492 & 1.7629 & 3.5961 & 1.4574 & 3.0767 \\
\hline \multirow[t]{6}{*}{2.0} & 0.4 & 1.8322 & 5.2176 & 1.8440 & 5.2733 & 1.8503 & 5.2983 \\
\hline & 0.6 & 1.7260 & 4.6855 & 1.7442 & 4.7835 & 1.7563 & 4.8321 \\
\hline & 0.8 & 1.6352 & 4.2020 & 1.6551 & 4.3386 & 1.6733 & 4.4121 \\
\hline & 1.5 & 1.4620 & 3.0364 & 1.4309 & 3.2239 & 1.4664 & 3.3814 \\
\hline & 2.0 & 1.4564 & 2.6068 & 1.3301 & 2.7152 & 1.3700 & 2.9254 \\
\hline & 4.0 & 2.0958 & 3.6392 & 1.1838 & 1.6496 & 1.1546 & 1.9922 \\
\hline \multirow[t]{6}{*}{4.0} & 0.4 & 1.7344 & 4.8228 & 1.7559 & 4.9001 & 1.7627 & 4.9251 \\
\hline & 0.6 & 1.5605 & 4.0589 & 1.6019 & 4.1951 & 1.6151 & 4.2403 \\
\hline & 0.8 & 1.4043 & 3.3984 & 1.4675 & 3.5897 & 1.4874 & 3.6530 \\
\hline & 1.5 & 1.0049 & 1.9031 & 1.1406 & 2.2381 & 1.1833 & 2.3432 \\
\hline & 2.0 & 0.8155 & 1.2870 & 0.9925 & 1.7112 & 1.0500 & 1.8310 \\
\hline & 4.0 & 0.4669 & -0.1633 & 0.6666 & 0.8077 & 0.7728 & 0.9349 \\
\hline \multirow[t]{6}{*}{6.0} & 0.4 & 1.7069 & 4.7081 & 1.7246 & 4.7731 & 1.7310 & 4.7964 \\
\hline & 0.6 & 1.5197 & 3.8941 & 1.5522 & 4.0017 & 1.5641 & 4.0428 \\
\hline & 0.8 & 1.3558 & 3.2107 & 1.4034 & 3.3521 & 1.4207 & 3.4078 \\
\hline & 1.5 & 0.9572 & 1.7788 & 1.0513 & 1.9565 & 1.0843 & 2.0406 \\
\hline & 2.0 & 0.7743 & 1.2870 & 0.8984 & 1.4383 & 0.9394 & 1.5299 \\
\hline & 4.0 & 0.3102 & 0.6548 & 0.5863 & 0.5921 & 0.6444 & 0.6837 \\
\hline
\end{tabular}

positive, which indicates that the function $h(x)$ is monotonically decreasing. Similarly, we analytically evaluate $d(x)$ for small values of $\alpha>1$ and the difference $d(x)$ is always negative. Numerically, we use the values of $q$ from 0.1 to 0.9 at an increment of 0.1 with values of $\alpha$ from 1.5 to 10.0 at an increment of 0.5 . All the $d(x)$ values are negative which indicates that the function $h(x)$ is monotonically increasing. Thus, we have a decreasing 
hazard rate when $\alpha<1$ and an increasing hazard rate when $\alpha>1$. For $\alpha=1, L_{1}=$ $L_{2}$ and we have a constant hazard rate. The graphs of the hazard rate function defined in Equation (24) are shown in Figure 2 for various values of the parameters. We see in Figure 2 that the hazard rate decreases for values of $\alpha<1$ and increases for $\alpha>1$.

The entropy of a random variable is a measure of variation of uncertainty. For a discrete random variable $X$ with probability mass function $g(x)$, the Shannon entropy is defined as,

$$
\mathcal{S}(x)=-\sum_{x} g(x) \log _{2} g(x) \geq 0 .
$$

In probabilistic context, $\mathcal{S}(x)$ is a measure of the information carried by $g(x)$, with higher entropy corresponding to less information. Substituting Equation (10) in Equation (25), we have

$$
\begin{aligned}
\mathcal{S}(x)= & -\sum_{x}\left\{\left[1-\left(1-q^{x}\right)^{\alpha}\right]^{\beta}-\left[1-\left(1-q^{x+1}\right)^{\alpha}\right]^{\beta}\right\} \\
& \times \log _{2}\left\{\left[1-\left(1-q^{x}\right)^{\alpha}\right]^{\beta}-\left[1-\left(1-q^{x+1}\right)^{\alpha}\right]^{\beta}\right\} .
\end{aligned}
$$

Suppose we write the PMF as

$$
\left[1-\left(1-q^{x}\right)^{\alpha}\right]^{\beta}\left\{1-\left(\frac{1-\left(1-q^{x+1}\right)^{\alpha}}{1-\left(1-q^{x}\right)^{\alpha}}\right)^{\beta}\right\} .
$$
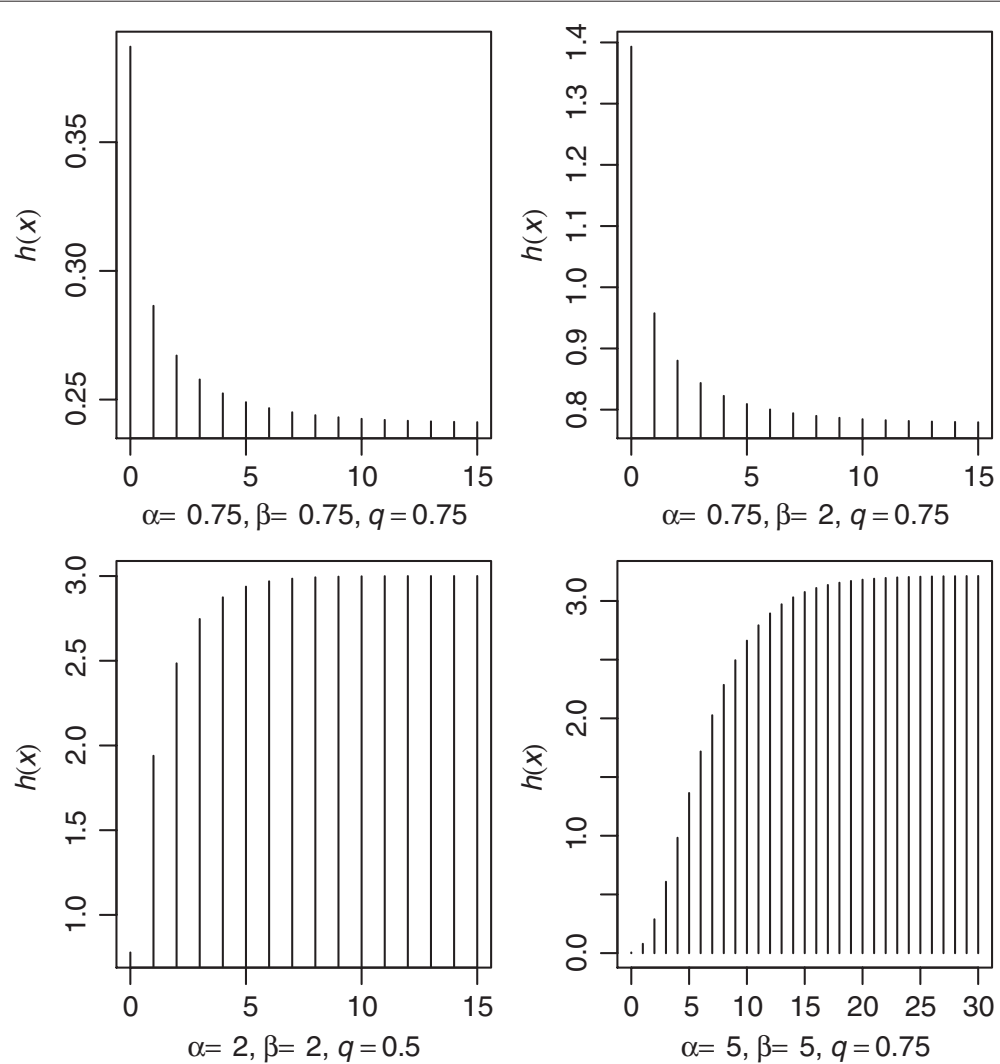

Figure 2 Hazard functions for values of $\alpha, \beta$ and $q$ 
Let $\alpha=1$ for simplicity. We may now write the entropy as

$$
\mathcal{S}(x)=-\sum_{x=0}^{\infty}\left(1-q^{\beta}\right) q^{x \beta} \log _{2}\left\{\left(1-q^{\beta}\right) q^{x \beta}\right\} .
$$

After some algebra, Equation (26) becomes,

$$
\mathcal{S}(x)=\frac{-\left(1-q^{\beta}\right) \log _{2}\left(1-q^{\beta}\right)-q^{\beta} \log _{2} q^{\beta}}{1-q^{\beta}}>0 .
$$

On setting $\beta=1$ in Equation (27), we have, for the geometric distribution,

$$
\mathcal{S}(x)=-\frac{q}{1-q} \log _{2} q-\log _{2}(1-q)=\frac{-(1-p) \log _{2}(1-p)-p \log _{2} p}{p} .
$$

Note that when $\beta=1, p=q=1 / 2, \mathcal{S}(x)=2$. It is not difficult to show that $\mathcal{S}(x)$ is an increasing function of $q$ for any given $\beta$. This is consistent with the pattern of the standard deviation. We also note that $\lim _{\beta \rightarrow \infty} \mathcal{S}(x)=0$, with the proviso that $0 \log 0=0$. This indicates that smaller values of $\beta$ increase the uncertainty in the distribution, while higher values of $\beta$ increase the amount of information measured in terms of the probability. Actually, a zero entropy indicates that all information needed is measured solely in terms of the probability. In a way, the KGD has smaller entropy (more probabilistic information) than the geometric distribution for values of $\beta>1$.

\section{Maximum likelihood estimation}

We discuss the maximum likelihood estimation of the parameters of the KGD in subsection 6.1. Subsection 6.2 contains the results of a simulation that is conducted to evaluate the performance of the maximum likelihood estimation method.

\subsection{Estimation}

Let a random sample of size $n$ be taken from KGD, with observed frequencies $n_{x}, x=$ $0,1,2, \ldots, k$, where $\sum_{x=0}^{k} n_{x}=n$. From Equation (10), the likelihood function for a random sample of size $n$ may be expressed as

$$
L(x \mid \alpha, \beta, q)=\prod_{x=0}^{k}\left\{\left[1-\left(1-q^{x}\right)^{\alpha}\right]^{\beta}-\left[1-\left(1-q^{x+1}\right)^{\alpha}\right]^{\beta}\right\}^{n_{x}}
$$

The log-likelihood function is

$$
\begin{aligned}
l(\alpha, \beta, q)= & \ln L(x \mid \alpha, \beta, q)=n_{0} \ln \left\{1-\left[1-(1-q)^{\alpha}\right]^{\beta}\right\} \\
& +\sum_{x=1}^{k} n_{x} \ln \left\{\left[1-\left(1-q^{x}\right)^{\alpha}\right]^{\beta}-\left[1-\left(1-q^{x+1}\right)^{\alpha}\right]^{\beta}\right\} .
\end{aligned}
$$


Differentiating the log-likelihood function with respect to the parameters, we obtain

$$
\begin{aligned}
\frac{\partial l(\alpha, \beta, q)}{\partial \alpha}= & \frac{n_{0} \beta(1-q)^{\alpha}\left[1-(1-q)^{\alpha}\right]^{\beta-1} \ln (1-q)}{1-\left[1-(1-q)^{\alpha}\right]^{\beta}} \\
& +\sum_{x=1}^{k} \frac{n_{x}\left[A_{x}+B_{x}\right]}{\left\{1-\left(1-q^{x}\right)^{\alpha}\right\}^{\beta}-\left\{1-\left(1-q^{x+1}\right)^{\alpha}\right\}^{\beta}}, \\
\frac{\partial l(\alpha, \beta, q)}{\partial \beta}= & \frac{-n_{0}\left[1-(1-q)^{\alpha}\right]^{\beta} \ln \left[1-(1-q)^{\alpha}\right]}{1-\left[1-(1-q)^{\alpha}\right]^{\beta}} \\
& +\sum_{x=1}^{k} \frac{n_{x}\left[C_{x}-D_{x}\right]}{\left\{1-\left(1-q^{x}\right)^{\alpha}\right\}^{\beta}-\left\{1-\left(1-q^{x+1}\right)^{\alpha}\right\}^{\beta}}, \\
\frac{\partial l(\alpha, \beta, q)}{\partial q}= & \frac{-n_{0} \alpha \beta\left[1-(1-q)^{\alpha}\right]^{\beta-1}(1-q)^{\alpha-1}}{1-\left[1-(1-q)^{\alpha}\right]^{\beta}} \\
& +\sum_{x=1}^{k} \frac{n_{x}\left[E_{x}-F_{x}\right]}{\left\{1-\left(1-q^{x}\right)^{\alpha}\right\}^{\beta}-\left\{1-\left(1-q^{x+1}\right)^{\alpha}\right\}^{\beta}},
\end{aligned}
$$

where,

$$
\begin{aligned}
& A_{x}=-\beta\left[1-\left(1-q^{x}\right)^{\alpha}\right]^{\beta-1}\left(1-q^{x}\right)^{\alpha} \ln \left(1-q^{x}\right), \\
& B_{x}=\beta\left[1-\left(1-q^{x+1}\right)^{\alpha}\right]^{\beta-1}\left(1-q^{x+1}\right)^{\alpha} \ln \left(1-q^{x+1}\right), \\
& C_{x}=\left[1-\left(1-q^{x}\right)^{\alpha}\right]^{\beta} \ln \left[1-\left(1-q^{x}\right)^{\alpha}\right], \\
& D_{x}=\left[1-\left(1-q^{x+1}\right)^{\alpha}\right]^{\beta} \ln \left[1-\left(1-q^{x+1}\right)^{\alpha}\right], \\
& E_{x}=\alpha \beta x q^{x-1}\left[1-\left(1-q^{x}\right)^{\alpha}\right]^{\beta-1}\left(1-q^{x}\right)^{\alpha-1}, \\
& F_{x}=\alpha \beta(x+1) q^{x}\left[1-\left(1-q^{x+1}\right)^{\alpha}\right]^{\beta-1}\left(1-q^{x+1}\right)^{\alpha-1} .
\end{aligned}
$$

Setting the non-linear Equations (30), (31) and (32) to zero and solving them iteratively, we get the estimates $\hat{\theta}=(\hat{\alpha}, \hat{\beta}, \hat{q})^{T}$ for the parameter vector $\theta=(\alpha, \beta, q)^{T}$. The initial values of parameters $\alpha$ and $\beta$ can be set to 1 and that of parameter $q$ can be set to 0.5 .

For interval estimation and hypothesis tests on the parameters, we require the information matrix $\mathcal{I}(\theta)$, with elements $-\partial^{2} l /(\partial i \partial j)=-l_{i j}$, where, $i, j \in\{\alpha, \beta, q\}$. Under conditions that are fulfilled for parameters in the interior of the parameter space but not on the boundary, the asymptotic distribution of $\sqrt{n}(\hat{\theta}-\theta)$ is $N_{3}\left(0, \mathcal{I}^{-1}(\theta)\right)$. The asymptotic multivariate normal distribution $N_{3}\left(0, \mathcal{I}^{-1}(\hat{\theta})\right)$ of $\hat{\theta}$ can be used to construct approximate confidence intervals for the parameters. For example, the $100(1-\xi) \%$ asymptotic confidence interval for the $i^{t h}$ parameter $\theta_{i}$ is given by

$$
\left(\hat{\theta}_{i}-z_{\xi / 2} \sqrt{\mathcal{I}_{i, i}}, \quad \hat{\theta}_{i}+z_{\xi / 2} \sqrt{\mathcal{I}_{i, i}}\right)
$$

where $\mathcal{I}_{i, i}$ is the $i^{t h}$ diagonal element of $\mathcal{I}^{-1}(\theta)$ for $i=1,2,3$, and $z_{\xi / 2}$ is the upper $\xi / 2$ point of standard normal distribution. See for example, Mahmoudi (2011). 
The information matrix $\mathcal{I}(\theta)$ is of the form,

$$
\mathcal{I}(\alpha, \beta, q)=\left(\begin{array}{lll}
l_{\alpha \alpha} & l_{\alpha \beta} & l_{\alpha q} \\
l_{\beta \alpha} & l_{\beta \beta} & l_{\beta q} \\
l_{q \alpha} & l_{q \beta} & l_{q q}
\end{array}\right) .
$$

The expressions for the elements $l_{i j}$ are given in the Appendix.

\subsection{Simulation}

A simulation study is conducted to evaluate the performance of the maximum likelihood estimation method. Equation (14) is used to generate a random sample from the KGD with parameters $\alpha, \beta$ and $q$. The different sample sizes considered in the simulation are $n=250,500$ and 750 . The parameter combinations for the simulation study are shown in Table 3. The combinations were chosen to reflect the following cases of the distribution: under-dispersion ( $\alpha=6, \beta=4, q=0.8$ ), over-dispersion (all other cases), monotonically decreasing ( $\alpha=1.6, \beta=2.0, q=0.6$ ), and unimodal with mode greater than 0 (all other cases). For each parameter combination and each sample size, the simulation process is repeated 100 times. The average bias (actual - estimate) and standard deviation of the parameter estimates are reported in Table 3. The biases are relatively small when compared to the standard deviations. In most cases, as the sample size increases, the standard deviations of the estimators decrease.

\section{Applications of KGD}

We apply the KGD to two data sets. The first data set is the observed frequencies of the distribution of purchases of a brand $X$ breakfast cereals purchased by consumers over a period of time (Consul 1989). The other data set is the number of absences among shift-workers in a steel industry (Gupta and Ong 2004). Comparisons are made with the generalized negative binomial distribution (GNBD) defined by Jain and Consul (1971) and

Table 3 Bias and standard deviation for maximum likelihood estimates

\begin{tabular}{|c|c|c|c|c|c|c|c|}
\hline \multirow[b]{2}{*}{$n$} & \multicolumn{3}{|c|}{ Actual values } & \multicolumn{3}{|c|}{ Bias with standard deviation in parentheses } & \multirow[b]{2}{*}{ Mode } \\
\hline & $\alpha$ & $\beta$ & $q$ & $\hat{\alpha}$ & $\hat{\boldsymbol{\beta}}$ & $\hat{q}$ & \\
\hline \multirow[t]{5}{*}{250} & 1.6 & 2.0 & 0.6 & $-0.1295(0.2099)$ & $0.1128(0.4697)$ & $0.0409(0.0733)$ & 0 \\
\hline & 4.0 & 2.0 & 0.4 & $-0.3136(0.7844)$ & $0.1047(0.4617)$ & $0.0237(0.0698)$ & 1 \\
\hline & 4.0 & 2.0 & 0.6 & $-0.1977(0.5714)$ & $0.1021(0.4571)$ & $0.0198(0.0571)$ & 2 \\
\hline & 4.0 & 2.0 & 0.8 & $-0.2467(0.5521)$ & $0.0852(0.4020)$ & $0.0126(0.0293)$ & 4 \\
\hline & 6.0 & 4.0 & 0.8 & $-0.3931(0.8570)$ & $0.2084(0.9507)$ & $0.0121(0.0278)$ & 5 \\
\hline \multirow[t]{5}{*}{500} & 1.6 & 2.0 & 0.6 & $-0.0437(0.1436)$ & $0.0121(0.4496)$ & $0.0153(0.0665)$ & 0 \\
\hline & 4.0 & 2.0 & 0.4 & $-0.3142(0.5876)$ & $0.1383(0.4421)$ & $0.0290(0.0639)$ & 1 \\
\hline & 4.0 & 2.0 & 0.6 & $-0.2015(0.4728)$ & $0.0808(0.4504)$ & $0.0194(0.0527)$ & 2 \\
\hline & 4.0 & 2.0 & 0.8 & $-0.2747(0.4630)$ & $0.1526(0.3781)$ & $0.0157(0.0278)$ & 4 \\
\hline & 6.0 & 4.0 & 0.8 & $-0.3283(0.7771)$ & $0.2187(0.9375)$ & $0.0116(0.0267)$ & 5 \\
\hline \multirow[t]{5}{*}{750} & 1.6 & 2.0 & 0.6 & $-0.0533(0.1305)$ & $0.1184(0.4250)$ & $0.0278(0.0669)$ & 0 \\
\hline & 4.0 & 2.0 & 0.4 & $-0.3181(0.5939)$ & $0.1707(0.4195)$ & $0.0328(0.0622)$ & 1 \\
\hline & 4.0 & 2.0 & 0.6 & $-0.1787(0.4775)$ & $0.0562(0.4469)$ & $0.0159(0.0522)$ & 2 \\
\hline & 4.0 & 2.0 & 0.8 & $-0.1717(0.4739)$ & $0.0813(0.4117)$ & $0.0101(0.0299)$ & 4 \\
\hline & 6.0 & 4.0 & 0.8 & $-0.2472(0.5940)$ & $0.1947(0.8090)$ & $0.0087(0.0227)$ & 5 \\
\hline
\end{tabular}


the exponentiated-exponential geometric distribution (EEGD) defined by Alzaatreh et al. (2012b).

\subsection{Purchases by consumers}

Consul (1989), p. 128 stated that, "The number of units of different commodities purchased by consumers over a period of time", appears to follow the generalized Poisson distribution (GPD). In support of this assertion, the author analyzed some relevant data sets and observed that the GPD model provided adequate fits. One of the data sets considered by Consul (1989) consists of observed frequencies of the distribution of purchases of a brand $X$ breakfast cereals. The original data in Table 4 was taken from Chatfield (1975).

The data contains the frequency of consumers who bought $r$ units of brand $X$ over a number of weeks. The data is fitted to the KGD, EEGD, and the GNBD. We are not sure how the frequencies for the (10-11), (12-15) and (16-35) were handled in previous applications of the data set. In our analysis, the probabilities for the classes (10-11) and (12-15) were obtained by adding the corresponding individual probabilities in each class. When finding the maximum likelihood estimates, the probability for the last class was obtained by subtracting the sum of all previous probabilities from 1 . The results in Table 4 show that all the three distributions provide adequate fit to the data. Since the parameter $\beta$ in KGD is not significantly different from 1 , it may be more appropriate to apply the two-parameter EEGD to fit the data. Also, the likelihood ratio test to compare the EEGD with the KGD is not significant at $5 \%$ level.

Table 4 The number of units $r$ purchased by observed number $\left(f_{r}\right)$ of consumers

\begin{tabular}{|c|c|c|c|c|}
\hline \multirow[b]{2}{*}{$r$-value } & \multirow[b]{2}{*}{ Observed } & \multicolumn{3}{|c|}{ Expected } \\
\hline & & EEGD & GNBD & KGD \\
\hline 0 & 299 & 300.11 & 298.98 & 299.33 \\
\hline 1 & 69 & 63.10 & 68.03 & 65.59 \\
\hline 2 & 37 & 41.13 & 41.12 & 42.02 \\
\hline 3 & 34 & 30.68 & 29.89 & 30.92 \\
\hline 4 & 23 & 24.36 & 23.51 & 24.28 \\
\hline 5 & 20 & 20.06 & 19.32 & 19.81 \\
\hline 6 & 12 & 16.92 & 16.32 & 16.58 \\
\hline 7 & 18 & 14.52 & 14.04 & 14.14 \\
\hline 8 & 14 & 12.61 & 12.25 & 12.21 \\
\hline 9 & 9 & 11.06 & 10.80 & 10.67 \\
\hline $10-11$ & 17 & 18.47 & 18.16 & 17.72 \\
\hline $12-15$ & 27 & 26.69 & 26.63 & 25.50 \\
\hline $16-35$ & 63 & 62.29 & 62.95 & 63.23 \\
\hline Total & 642 & 642 & 642 & 642 \\
\hline Parameter & & $\hat{\alpha}=0.2910(0.0212)$ & $\hat{\theta}=0.9658(0.0148)$ & $\hat{\alpha}=0.3373(0.0924)$ \\
\hline \multirow[t]{2}{*}{ Estimates } & & $\hat{\theta}=0.9267(0.0076)$ & $\hat{m}=0.2264(0.0339)$ & $\hat{\beta}=1.5114(1.3648)$ \\
\hline & & & $\hat{\beta}=0.9882(0.0126)$ & $\hat{q}=0.9592(0.0544)$ \\
\hline$\overline{\chi^{2}}$ & & 4.34 & 3.92 & 4.11 \\
\hline$d f$ & & 10 & 9 & 9 \\
\hline$p$-value & & 0.9307 & 0.9166 & 0.9040 \\
\hline $\mathrm{AIC}$ & & 2471.3 & 2472.8 & 2473.0 \\
\hline $\mathrm{LL}^{*}$ & & -1233.64 & -1233.38 & -1233.50 \\
\hline
\end{tabular}




\subsection{Number of absences by shift-workers}

The KGD is also applied to a data set from Gupta and Ong (2004), which represents the observed frequencies of the number of absences among shift-workers in a steel industry. The data in Table 5 was originally studied by Arbous and Sichel (1954) in an attempt to create a model that can describe the distribution of absences to a group of people in single- and double-exposure periods. The original data contains the number of absences, $x$-value, of 248 shift workers in the years 1947 and 1948. Arbous and Sichel (1954) used the negative binomial distribution (NBD) to fit the data. Gupta and Ong (2004) proposed a four-parameter generalized negative binomial distribution to fit the data and compared it to the NBD and the GPD. The chi-square value for their distribution was 8.27 with 15

Table $\mathbf{5}$ The number of absences among shift-workers in a steel industry

\begin{tabular}{|c|c|c|c|c|}
\hline \multirow[b]{2}{*}{$x$-value } & \multirow{2}{*}{$\begin{array}{l}\text { Observed } \\
\text { Frequency }\end{array}$} & \multicolumn{3}{|c|}{ Expected } \\
\hline & & EEGD & GNBD & KGD \\
\hline 0 & 7 & 10.18 & 9.86 & 6.52 \\
\hline 1 & 16 & 16.41 & 16.44 & 18.21 \\
\hline 2 & 23 & 18.30 & 19.32 & 21.40 \\
\hline 3 & 20 & 18.57 & 19.84 & 20.92 \\
\hline 4 & 23 & 18.02 & 19.09 & 19.31 \\
\hline 5 & 24 & 17.03 & 17.73 & 17.45 \\
\hline 6 & 12 & 15.82 & 16.13 & 15.64 \\
\hline 7 & 13 & 14.52 & 14.50 & 13.97 \\
\hline 8 & 9 & 13.22 & 12.93 & 12.46 \\
\hline 9 & 9 & 11.95 & 11.49 & 11.11 \\
\hline 10 & 8 & 10.74 & 10.17 & 9.90 \\
\hline 11 & 10 & 9.62 & 9.00 & 8.83 \\
\hline 12 & 8 & 8.59 & 7.95 & 7.87 \\
\hline 13 & 7 & 7.65 & 7.02 & 7.01 \\
\hline 14 & $2^{a}$ & 6.79 & 6.21 & 6.25 \\
\hline 15 & $12^{a}$ & 6.02 & 5.49 & 5.57 \\
\hline 16 & $3^{b}$ & 5.33 & 4.86 & 4.96 \\
\hline 17 & $5^{b}$ & 4.71 & 4.30 & 4.42 \\
\hline 18 & $4^{c}$ & 4.16 & 3.81 & 3.94 \\
\hline 19 & $2^{c}$ & 3.67 & 3.38 & 3.51 \\
\hline 20 & $2^{d}$ & 3.23 & 3.00 & 3.13 \\
\hline 21 & $5^{d}$ & 2.85 & 2.67 & 2.79 \\
\hline 22 & $5^{e}$ & 2.51 & 2.38 & 2.49 \\
\hline 23 & $2^{e}$ & 2.21 & 2.11 & 2.21 \\
\hline 24 & $1^{e}$ & 1.94 & 1.88 & 1.97 \\
\hline $25-48$ & 16 & 13.96 & 16.44 & 16.16 \\
\hline Total & 248 & 248 & 248 & 248 \\
\hline Parameter & & $\hat{\alpha}=1.5255(0.1471)$ & $\hat{\theta}=0.0026(0.0001)$ & $\hat{\alpha}=3.1859(1.6702)$ \\
\hline Estimates & & $\hat{\theta}=0.8767(0.0093)$ & $\hat{m}=1242.96(4.259)$ & $\hat{\beta}=0.1292(0.0882)$ \\
\hline & & & $\hat{\beta}=254.77(20.785)$ & $\hat{q}=0.4099(0.2374)$ \\
\hline$\overline{\chi^{2}}$ & & 12.18 & 9.62 & 7.78 \\
\hline$d f$ & & 17 & 16 & 16 \\
\hline$p$-value & & 0.7891 & 0.8857 & 0.9551 \\
\hline$\overline{A I C}$ & & 1517.8 & 1517.3 & 1515.2 \\
\hline Log-likelihood & & -756.88 & -755.66 & -754.62 \\
\hline
\end{tabular}


degrees of freedom. The chi-square value obtained by Gupta and Ong for the GPD was 27.79 with 17 degrees of freedom (DF). The DF $=k-s-1$, where $k$ is the number of classes and $s$ is the number of estimated parameters.

We re-analyzed the data for the EEGD, the GNBD, and the GPD. We obtained a chisquare of 9.62 for the GPD, which is much smaller than the 27.79 provided by Gupta and Ong. Thus, our estimates from the GPD (not reported in Table 5) differ significantly from the results in Gupta and Ong (2004). When finding the maximum likelihood estimates, the probability for the last class was obtained by subtracting the sum of all previous probabilities from 1. In view of this, the results obtained from the EEGD are slightly different from those of Alzaatreh et al. (2012b) who applied the EEGD to fit the data. We apply the KGD to model the data in Table 5, and the results from the table indicate that the KGD, EEGD and GNBD provide good fit to the data.

If $m \rightarrow \infty$, the GNBD with parameters $\theta, m$ and $\beta$ goes to the GPD with parameters $\alpha$ and $\lambda$, where $\alpha=m \theta$ and $\lambda=\theta \beta$ on page 218 of Consul and Famoye (2006). We fitted the GPD to the data and we got the same log-likelihood with $\hat{\alpha}=3.2250$ and $\hat{\lambda}=0.6597$. From the GNBD, we obtained $\hat{m} \hat{\theta}=1242.96 \times 0.002591=3.22 \approx 3.2250=\hat{\alpha}$ and $\hat{\beta} \hat{\theta}=254.77 \times 0.002591=0.66 \approx 0.6597=\hat{\lambda}$.

We observe that the parameter $\beta$ in the KGD is significantly different from 1 . This makes the KGD a more appropriate distribution over the EEGD. The likelihood ratio statistic for testing the EEGD against the KGD is $\chi_{1}^{2}=-2(754.62-756.88)=4.52$ with a $p$-value of 0.0335 . Thus, we reject the null hypothesis that the data follows the EEGD at the $5 \%$ level. The likelihood ratio test supports the claim that the parameter $\beta$ is significantly different from 1 , and hence the KGD appears to be superior to the EEGD.

\section{Conclusion}

Discrete distributions are often derived by using the Lagrange expansions framework (see for example Consul and Famoye 2006) or using difference equations (see for example Johnson et al. 2005). Recently, Alzaatreh et al. (2012b, 2013b) developed a general method for generating distributions and these distributions are members of the $T-X$ family. The method can be applied to derive both the discrete and continuous distributions. This article used the $T-X$ family framework to define a new discrete distribution named the Kumaraswamy-geometric distribution (KGD).

Some special cases, and properties of the KGD are discussed, which include moments, hazard rate and entropy. The method of maximum likelihood estimation is used in estimating the parameters of the KGD. The distribution is applied to model two real life data sets; one consisting of the observed frequencies of the distribution of purchases of a brand $X$ breakfast cereals, and the other, the observed frequencies of the number of absences among shift-workers in a steel industry. Two other distributions, the EEGD and the GNBD are compared with KGD. It is found that the KGD performed as well as the EEGD in modeling the observed numbers of consumers. The results also show that the KGD outperformed the EEGD in modeling the number of absences among shift-workers. It is expected that the additional parameter offered by the Kumaraswamy's distribution will enable the use of the KGD in modeling events where the EEGD or the geometric distribution may not provide adequate fits. 


\section{Appendix}

\section{Elements of the information matrix}

$$
\begin{aligned}
& l_{\alpha \alpha}=\frac{A_{0}\left[1-\beta(1-q)^{\alpha}\right] \ln (1-q)}{P_{0}\left[1-(1-q)^{\alpha}\right]}-\frac{A_{0} B_{0}}{P_{0}^{2}}+\sum_{x=1}^{k} \frac{n_{x}}{P_{x}}\left[\frac{\partial\left(A_{x}+B_{x}\right)}{\partial \alpha}-\frac{\left(A_{x}+B_{x}\right)^{2}}{P_{x}}\right] \text {, } \\
& l_{\alpha \beta}=\frac{A_{0}}{P_{0} \beta}+\frac{A_{0} \ln \left[1-(1-q)^{\alpha}\right]}{P_{0}^{2}}+\sum_{x=1}^{k} \frac{n_{x}}{P_{x}}\left[\frac{\partial\left(A_{x}+B_{x}\right)}{\partial \beta}-\frac{\left(A_{x}+B_{x}\right)\left(C_{x}-D_{x}\right)}{P_{x}}\right] \text {, } \\
& l_{\alpha q}=\frac{A_{0}}{P_{0}}\left[\frac{\alpha\left\{\beta(1-q)^{\alpha}-1+\left[1-(1-q)^{\alpha}\right]^{\beta}\right\}}{(1-q)\left[1-(1-q)^{\alpha}\right] P_{0}}-\frac{1}{(1-q) \ln (1-q)}\right] \\
& +\sum_{x=1}^{k} \frac{n_{x}}{P_{x}}\left[\frac{\partial\left(A_{x}+B_{x}\right)}{\partial q}-\frac{\left(A_{x}+B_{x}\right)\left(E_{x}-F_{x}\right)}{P_{x}}\right], \\
& l_{\beta \beta}=\frac{C_{0} \ln \left[1-(1-q)^{\alpha}\right]}{P_{0}^{2}}+\sum_{x=1}^{k} \frac{n_{x}}{P_{x}}\left[\frac{\partial\left(C_{x}-D_{x}\right)}{\partial \beta}-\frac{\left(C_{x}-D_{x}\right)^{2}}{P_{x}}\right] \text {, } \\
& l_{\beta q}=\frac{C_{0}}{P_{0}}\left[\frac{\alpha \beta(1-q)^{\alpha-1}}{\left[1-(1-q)^{\alpha}\right] P_{0}}+\frac{\alpha(1-q)^{\alpha-1}}{\left[1-(1-q)^{\alpha}\right] \ln \left[1-(1-q)^{\alpha}\right]}\right] \\
& +\sum_{x=1}^{k} \frac{n_{x}}{P_{x}}\left[\frac{\partial\left(C_{x}-D_{x}\right)}{\partial q}-\frac{\left(C_{x}-D_{x}\right)\left(E_{x}-F_{x}\right)}{P_{x}}\right] \text {, } \\
& l_{q q}=\frac{E_{0}}{P_{0}}\left[\frac{\alpha \beta(1-q)^{\alpha-1}\left[1-(1-q)^{\alpha}\right]^{\beta-1}}{P_{0}}+\frac{(\alpha \beta-1)(1-q)^{\alpha}-\alpha+1}{(1-q)\left[1-(1-q)^{\alpha}\right]}\right] \\
& +\sum_{x=1}^{k} \frac{n_{x}}{P_{x}}\left[\frac{\partial\left(E_{x}-F_{x}\right)}{\partial q}-\frac{\left(E_{x}-F_{x}\right)^{2}}{P_{x}}\right] \text {, }
\end{aligned}
$$

where,

$$
\begin{aligned}
P_{x} & =\left[1-\left(1-q^{x}\right)^{\alpha}\right]^{\beta}-\left[1-\left(1-q^{x+1}\right)^{\alpha}\right]^{\beta}, \\
P_{0} & =1-\left[1-(1-q)^{\alpha}\right]^{\beta} \\
A_{0} & =n_{0} \beta(1-q)^{\alpha}\left[1-(1-q)^{\alpha}\right]^{\beta-1} \ln (1-q), \\
B_{0} & =\beta(1-q)^{\alpha}\left[1-(1-q)^{\alpha}\right]^{\beta-1} \ln (1-q), \\
C_{0} & =-n_{0}\left[1-(1-q)^{\alpha}\right]^{\beta} \ln \left[1-(1-q)^{\alpha}\right], \\
E_{0} & =-n_{0} \alpha \beta\left[1-(1-q)^{\alpha}\right]^{\beta-1}(1-q)^{\alpha-1}, \\
\frac{\partial A_{x}}{\partial \alpha} & =\frac{A_{x}\left[1-\beta\left(1-q^{x}\right)^{\alpha}\right] \ln \left(1-q^{x}\right)}{1-\left(1-q^{x}\right)^{\alpha}}, \\
\frac{\partial A_{x}}{\partial \beta} & =A_{x}\left(1 / \beta+\ln \left[1-\left(1-q^{x}\right)^{\alpha}\right]\right), \\
\frac{\partial A_{x}}{\partial q} & =\frac{A_{x} \alpha x q^{x-1}\left[\beta\left(1-q^{x}\right)^{\alpha}-1\right]}{\left(1-q^{x}\right)\left[1-\left(1-q^{x}\right)^{\alpha}\right]}-\frac{A_{x} x q^{x-1}}{\left(1-q^{x}\right) \ln \left(1-q^{x}\right)} \\
\frac{\partial B_{x}}{\partial \alpha} & =\frac{B_{x}\left[1-\beta\left(1-q^{x+1}\right)^{\alpha}\right] \ln \left(1-q^{x+1}\right)}{1-\left(1-q^{x+1}\right)^{\alpha}}, \\
\frac{\partial B_{x}}{\partial \beta} & =B_{x}\left(1 / \beta+\ln \left[1-\left(1-q^{x+1}\right)^{\alpha}\right]\right),
\end{aligned}
$$




$$
\begin{aligned}
& \frac{\partial B_{x}}{\partial q}=\frac{B_{x} \alpha(x+1) q^{x}\left[\beta\left(1-q^{x+1}\right)^{\alpha}-1\right]}{\left(1-q^{x+1}\right)\left[1-\left(1-q^{x+1}\right)^{\alpha}\right]}-\frac{B_{x}(x+1) q^{x}}{\left(1-q^{x+1}\right) \ln \left(1-q^{x+1}\right)}, \\
& \frac{\partial C_{x}}{\partial \alpha}=\frac{-C_{x}\left(1-q^{x}\right)^{\alpha} \ln \left(1-q^{x}\right)}{1-\left(1-q^{x}\right)^{\alpha}}\left(\beta+\frac{1}{\ln \left[1-\left(1-q^{x}\right)^{\alpha}\right]}\right) \text {, } \\
& \frac{\partial C_{x}}{\partial \beta}=C_{x} \ln \left[1-\left(1-q^{x}\right)^{\alpha}\right] \\
& \frac{\partial C_{x}}{\partial q}=\frac{C_{x} \alpha x q^{x-1}\left(1-q^{x}\right)^{\alpha-1}}{1-\left(1-q^{x}\right)^{\alpha}}\left(\beta+\frac{1}{\ln \left[1-\left(1-q^{x}\right)^{\alpha}\right]}\right), \\
& \frac{\partial D_{x}}{\partial \alpha}=\frac{-D_{x}\left(1-q^{x+1}\right)^{\alpha} \ln \left(1-q^{x+1}\right)}{1-\left(1-q^{x+1}\right)^{\alpha}}\left(\beta+\frac{1}{\ln \left[1-\left(1-q^{x+1}\right)^{\alpha}\right]}\right) \text {, } \\
& \frac{\partial D_{x}}{\partial \beta}=D_{x} \ln \left[1-\left(1-q^{x+1}\right)^{\alpha}\right] \text {, } \\
& \frac{\partial D_{x}}{\partial q}=\frac{D_{x} \alpha(x+1) q^{x}\left(1-q^{x+1}\right)^{\alpha-1}}{1-\left(1-q^{x+1}\right)^{\alpha}}\left(\beta+\frac{1}{\ln \left[1-\left(1-q^{x+1}\right)^{\alpha}\right]}\right) \text {, } \\
& \frac{\partial E_{x}}{\partial \alpha}=E_{x}\left(1 / \alpha+\frac{\left[1-\beta\left(1-q^{x}\right)^{\alpha}\right] \ln \left(1-q^{x}\right)}{1-\left(1-q^{x}\right)^{\alpha}}\right) \text {, } \\
& \frac{\partial E_{x}}{\partial \beta}=E_{x}\left(1 / \beta+\ln \left[1-\left(1-q^{x}\right)^{\alpha}\right]\right) \text {, } \\
& \frac{\partial E_{x}}{\partial q}=\frac{E_{x}(x-1)}{q}+\frac{E_{x}\left[(\alpha \beta-1)\left(1-q^{x}\right)^{\alpha}-\alpha+1\right] x q^{x-1}}{\left(1-q^{x}\right)\left[1-\left(1-q^{x}\right)^{\alpha}\right]}, \\
& \frac{\partial F_{x}}{\partial \alpha}=F_{x}\left(1 / \alpha+\frac{\left[1-\beta\left(1-q^{x+1}\right)^{\alpha}\right] \ln \left(1-q^{x+1}\right)}{1-\left(1-q^{x+1}\right)^{\alpha}}\right), \\
& \frac{\partial F_{x}}{\partial \beta}=F_{x}\left(1 / \beta+\ln \left[1-\left(1-q^{x+1}\right)^{\alpha}\right]\right) \text {, and } \\
& \frac{\partial F_{x}}{\partial q}=\frac{F_{x} x}{q}+\frac{F_{x}\left[(\alpha \beta-1)\left(1-q^{x+1}\right)^{\alpha}-\alpha+1\right](x+1) q^{x}}{\left(1-q^{x+1}\right)\left[1-\left(1-q^{x+1}\right)^{\alpha}\right]} .
\end{aligned}
$$

The values of $A_{x}, B_{x}, C_{x}, D_{x}, E_{x}$, and $F_{x}$ are given in Section 6.

\section{Competing interests}

The authors declare that they have no competing interests.

\section{Authors' contributions}

The authors, viz AA, FF and CL with the consultation of each other carried out this work and drafted the manuscript together. All authors read and approved the final manuscript.

\section{Acknowledgment}

The authors are grateful to the Associate Editor and the anonymous referees for many constructive comments and suggestions that have greatly improved the paper.

\section{Author details}

${ }^{1}$ Department of Mathematics, Marshall University, Huntington, West Virginia 25755, USA. ${ }^{2}$ Department of Mathematics, Central Michigan University, Mount Pleasant, Michigan 48859, USA.

Received: 4 February 2014 Accepted: 15 July 2014

Published online: 01 November 2014

\section{References}

Akinsete, A, Famoye, F, Lee, C: The beta-Pareto distributions. Statistics. 42(6), 547-563 (2008)

Alexander, C, Cordeiro, GM, Ortega, EMM, Sarabia, JM: Generalized beta-generated distributions. Comput. Stat. Data Anal. 56, 1880-1897 (2012)

Alshawarbeh, E, Famoye, F, Lee, C: Beta-Cauchy distribution: some properties and its applications. J. Stat. Theory Appl. 12, 378-391 (2013) 
Alzaatreh, A, Famoye, F, Lee, C: Weibull-Pareto distribution and its applications. Comm. Stat. Theor. Meth.

42(7), 1673-1691 (2013a)

Alzaatreh, A, Lee, C, Famoye, F: On the discrete analogues of continuous distributions. Stat. Meth. 9, 589-603 (2012b) Alzaatreh, A, Lee, C, Famoye, F: A new method for generating families of continuous distributions. Metron. 71, 63-79 (2013b)

Arbous, AG, Sichel, HS: New techniques for the analysis of asenteeism data. Biometrika. 41, 77-90 (1954)

Chatfield, C: A marketing application of characterization theorem. In: Patil, GP, Kotz, S, Ord, JK (eds.) Statistical Distributions in Scientific Work, volume 2, pp. 175-185. D. Reidel Publishing Company, Boston, (1975)

Consul, PC: Generalized Poisson Distributions: Properties and Applications. Marcel Dekker, Inc., New York (1989)

Consul, PC, Famoye, F: Lagrangian Probability Distributions. Birkhäuser, Boston (2006)

Cordeiro, GM, de Castro, M: A new family of generalized distributions. J. Stat. Comput. Simulat. 81(7), 883-898 (2011)

Cordeiro, GM, Lemonte, AJ: The beta Laplace distribution. Stat. Probability Lett. 81, 973-982 (2011)

Cordeiro, GM, Nadarajah, S, Ortega, EMM: The Kumaraswamy Gumbel distribution. Stat. Methods Appl. 21, 139-168 (2012)

de Pascoa, MAR, Ortega, EMM, Cordeiro, GM: The Kumaraswamy generalized gamma distribution with application in survival analysis. Stat. Meth. 8, 411-433 (2011)

de Santana, TV, Ortega, EMM, Cordeiro, GM, Silva, GO: The Kumaraswamy-log-logistic distribution. Stat. Theory Appl. 3, 265-291 (2012)

Eugene, N, Lee, C, Famoye, F: The beta-normal distribution and its applications. Comm. Stat. Theor. Meth. 31(4), 497-512 (2002)

Famoye, F, Lee, C, Olumolade, O: The beta-Weibull distribution. J. Stat. Theory Appl. 4(2), 121-136 (2005)

Gupta, RC, Ong, SD: A new generalization of the negative binomial distribution. Comput. Stat. Data Anal. 45, 287-300 (2004)

Jain, GC, Consul, PC: A generalized negative binomial distribution. SIAM J. Appl. Math. 21, 501-513 (1971)

Johnson, NL, Kemp, AW, Kotz, S: Univariate Discrete Distributiuons. third edition. John Wiley \& Sons, New York (2005)

Jones, MC: Kumaraswamy's distribution: a beta-type distribution with some tractability advantages. Stat. Methodologies. 6, 70-81 (2009)

Kong, L, Lee, C, Sepanski, JH: On the properties of of beta-gamma distribution. J. Mod. Appl. Stat. Meth. 6(1), 187-211 (2007)

Kumaraswamy, P: A generalized probability density function for double-bounded random processes. Hydrology 46, 79-88 (1980)

Lemonte, AJ, Barreto-Souza, W, Cordeiro, GM: The exponentiated Kumaraswamy distribution and its log-transform. Braz. J. Probability Stat. 27, 31-53 (2013)

Mahmoudi, E: The beta generalized Pareto distribution with application to lifetime data. Math. Comput. Simulations. 81, 2414-2430 (2011)

Nadarajah, S, Kotz, S: The beta Gumbel distribution. Math. Probl. Eng. 2004(4), 323-332 (2004)

Nadarajah, S, Kotz, S: The beta exponential distribution. Reliability Eng. Syst. Saf. 91, 689-697 (2006)

Singla, N, Jain, K, Sharma, SK: The beta generalized Weibull distribution: properties and applications. Reliability Eng. Syst. Saf. 102, 5-15 (2012)

Zwillinger, D, Kokoska, S: Standard Probability and Statistics Tables and Formulae. Chapman and Hall/CRC, Boca Raton (2000)

doi:10.1186/s40488-014-0017-1

Cite this article as: Akinsete et al:: The Kumaraswamy-geometric distribution. Journal of Statistical Distributions and

Applications 2014 1:17.

\section{Submit your manuscript to a SpringerOpen ${ }^{\circ}$ journal and benefit from:}

- Convenient online submission

- Rigorous peer review

- Immediate publication on acceptance

- Open access: articles freely available online

- High visibility within the field

- Retaining the copyright to your article

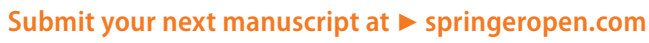

